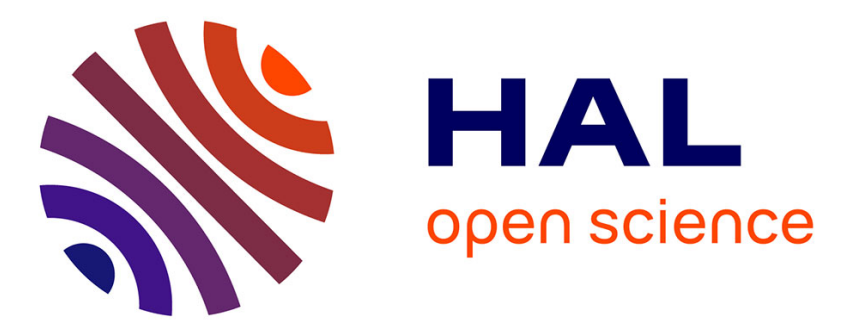

\title{
Strain mapping at the nanoscale using precession electron diffraction in transmission electron microscope with off axis camera
}

Matthieu M.P. Vigouroux, V. Delaye, N. N. Bernier, R. Cipro, D. Lafond, G. Audoit, T. Baron., J.L. Rouvière, M. Martin, Bernard Chenevier, et al.

\section{To cite this version:}

Matthieu M.P. Vigouroux, V. Delaye, N. N. Bernier, R. Cipro, D. Lafond, et al.. Strain mapping at the nanoscale using precession electron diffraction in transmission electron microscope with off axis camera. Applied Physics Letters, 2014, 105 (19), pp.191906. 10.1063/1.4901435 . hal-01103079

\section{HAL Id: hal-01103079 \\ https://hal.univ-grenoble-alpes.fr/hal-01103079}

Submitted on 14 Jan 2015

HAL is a multi-disciplinary open access archive for the deposit and dissemination of scientific research documents, whether they are published or not. The documents may come from teaching and research institutions in France or abroad, or from public or private research centers.
L'archive ouverte pluridisciplinaire HAL, est destinée au dépôt et à la diffusion de documents scientifiques de niveau recherche, publiés ou non, émanant des établissements d'enseignement et de recherche français ou étrangers, des laboratoires publics ou privés. 


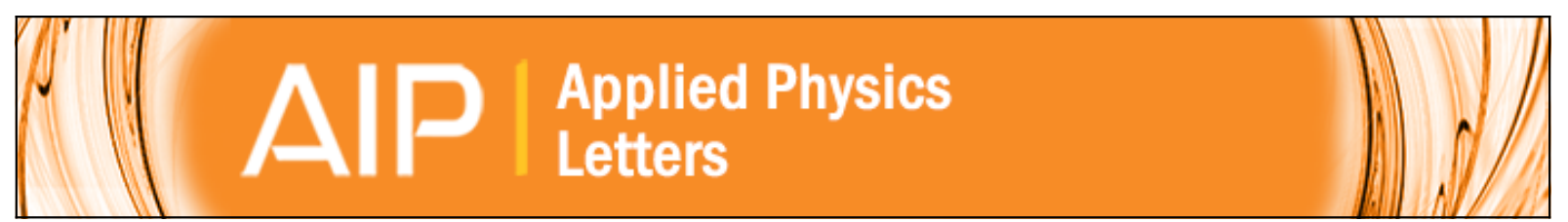

\section{Strain mapping at the nanoscale using precession electron diffraction in transmission} electron microscope with off axis camera

M. P. Vigouroux, V. Delaye, N. Bernier, R. Cipro, D. Lafond, G. Audoit, T. Baron, J. L. Rouvière, M. Martin, B. Chenevier, and F. Bertin

Citation: Applied Physics Letters 105, 191906 (2014); doi: 10.1063/1.4901435

View online: http://dx.doi.org/10.1063/1.4901435

View Table of Contents: http://scitation.aip.org/content/aip/journal/apl/105/19?ver=pdfcov

Published by the AIP Publishing

\section{Articles you may be interested in}

Improved strain precision with high spatial resolution using nanobeam precession electron diffraction Appl. Phys. Lett. 103, 241913 (2013); 10.1063/1.4829154

Improved precision in strain measurement using nanobeam electron diffraction

Appl. Phys. Lett. 95, 123114 (2009); 10.1063/1.3224886

Local strain measurement in a strain-engineered complementary metal-oxide-semiconductor device by geometrical phase analysis in the transmission electron microscope

Appl. Phys. Lett. 93, 081909 (2008); 10.1063/1.2970050

nlinlmproving spatial resolution of convergent beam electron diffraction strain mapping in silicon microstructures Appl. Phys. Lett. 86, 063508 (2005); 10.1063/1.1855408

Application of convergent beam electron diffraction to two-dimensional strain mapping in silicon devices Appl. Phys. Lett. 82, 2172 (2003); 10.1063/1.1565181 


\title{
Strain mapping at the nanoscale using precession electron diffraction in transmission electron microscope with off axis camera
}

\author{
M. P. Vigouroux, ${ }^{1,2}$ V. Delaye, ${ }^{1,2}$ N. Bernier, ${ }^{1,2}$ R. Cipro, ${ }^{1,3}$ D. Lafond, ${ }^{1,2}$ G. Audoit, ${ }^{1,2}$ \\ T. Baron, ${ }^{1,3}$ J. L. Rouvière, ${ }^{1,4}$ M. Martin, ${ }^{1,3}$ B. Chenevier, ${ }^{1,5}$ and F. Bertin ${ }^{1,2}$ \\ ${ }^{1}$ Université Grenoble Alpes, F-38000 Grenoble, France \\ ${ }^{2}$ CEA, LETI, MINATEC Campus, 17 rue des martyrs, 38054 GRENOBLE Cedex 9, France \\ ${ }^{3}$ CNRS, LTM, F-38000 Grenoble, France \\ ${ }^{4}$ CEA, INAC, MINATEC Campus, 17 rue des martyrs, 38054 GRENOBLE Cedex 9, France \\ ${ }^{5}$ LMGP, CNRS, 3 parvis Louis Néel, 38016 GRENOBLE Cedex 1, France
}

(Received 1 August 2014; accepted 29 October 2014; published online 12 November 2014)

\begin{abstract}
Precession electron diffraction is an efficient technique to measure strain in nanostructures by precessing the electron beam, while maintaining a few nanometre probe size. Here, we show that an advanced diffraction pattern treatment allows reproducible and precise strain measurements to be obtained using a default $512 \times 512$ DigiSTAR off-axis camera both in advanced or noncorrected transmission electron microscopes. This treatment consists in both projective geometry correction of diffraction pattern distortions and strain Delaunay triangulation based analysis. Precision in the strain measurement is improved and reached $2.7 \times 10^{-4}$ with a probe size approaching $4.2 \mathrm{~nm}$ in diameter. This method is applied to the study of the strain state in InGaAs quantum-well (QW) devices elaborated on Si substrate. Results show that the GaAs/Si mismatch does not induce in-plane strain fluctuations in the InGaAs QW region. (C) 2014 AIP Publishing LLC. [http://dx.doi.org/10.1063/1.4901435]
\end{abstract}

Precession Electron Diffraction (PED) ${ }^{1,2}$ is an innovative method for the crystallographic study of materials. The advantage of PED is to minimize the diffraction dynamical effects to such an extent that diffraction images can be analysed using a kinematical approach with minimal user intervention. Widely used to obtain precise orientation and phase mappings ${ }^{3}$ or solving crystal structures, ${ }^{4}$ PED can also be used to improve measurement of strain in nanostructures. ${ }^{5}$

In practice, one usual way of precessing the incident beam is performed with the widely used NanoMEGAS's "DigiSTAR" add-on device. ${ }^{7}$ Instead of working with the high-resolution on-axis CCD camera, this system is equipped with the high frame rate and high sensitivity Stingrey detection system that allows for fast mapping experiments. However, this camera is focused on the fluorescent screen, and it is therefore deported from the microscope optical axis. This off-axis camera induces significant perspective distortions in the measured diffraction patterns. As a consequence, the use of standard diffraction pattern treatment, e.g., a noise reduction filter and a two-dimensional Gaussian fitting of diffracted spots, ${ }^{8}$ may be inappropriate due to the loss of periodicity in the acquired patterns.

In this letter, we demonstrate that PED can be advantageously used in a TEM to map strain at nanoscale in an unexplored situation where the use of an off-axis camera requires an advanced diffraction pattern treatment. We propose here an alternative strain analysis able to find a "quasikinematic" disc shaped region containing strongly correlated spots, and a way to correct off-axis camera's distortions. We have performed PED strain measurements on a simple reference sample and in a more complex InGaAs structure for microelectronic applications.

PED measurements were made using a JEOLJEM2010FEF non-corrected TEM operating at $200 \mathrm{kV}$.
Precession beam scan alignment ${ }^{6}$ was performed employing NanoMEGAS's "DigiSTAR" add-on device. ${ }^{7}$ Precession semi-angle was set to $1.44^{\circ}$ to take full advantage of PED kinematical behaviour. With $10 \mu \mathrm{m}$ condenser aperture, a probe size as small as $4.2 \mathrm{~nm}$ full width at half maximum is obtained on the sample with a convergence semi-angle of $0.5 \mathrm{mrad}$.

PED biaxial strain measurements were made on the $\langle 110\rangle$ zone axis of samples. Each PED pattern is recorded every $2.7 \mathrm{~nm}$ in a $200 \mathrm{~nm} \times 240 \mathrm{~nm}$ area indicated in Fig. 2(a) using a $512 \times 512$ pixel Stingrey camera deported from the microscope optical. As mentioned above, the perspective effect induces a periodicity loss in the acquired images. In the present work, the periodicity of the diffraction pattern is retrieved using classical projective geometry. ${ }^{9}$

To this end, we maximize-using a simplex algorithm-the diffraction pattern power spectral density root mean square (rms) contras $\mathrm{t}^{10}$ by adjusting three parameters: the camera distance, the roll angle, and the off axis angle (the total spectral energy is set to unity all along this treatment). The three parameters are used to correct the whole PED data set. Figures 1(a) and 1(b) show raw and corrected silicon $\langle 110\rangle$ zone axis diffraction patterns, respectively. After projective geometry correction, the diffraction pattern of an amorphous phase (Figure 1(c)) only shows the residual astigmatism minimized by the user during the microscope alignment.

The two-dimensional fitting of the complete set of diffracted spots is the most widespread means to retrieve strain from diffraction patterns, as applied to nanobeam diffraction $(\mathrm{NBD})^{8,11}$ precession $^{5}$ or convergent beam electron diffraction $(\mathrm{CBED})^{8}$ experiments. In this paper, we propose an alternative method that results in an increase of the strain sensitivity (as shown in Table I). In contrast to standard methods that use the whole image (in real or Fourier space), 

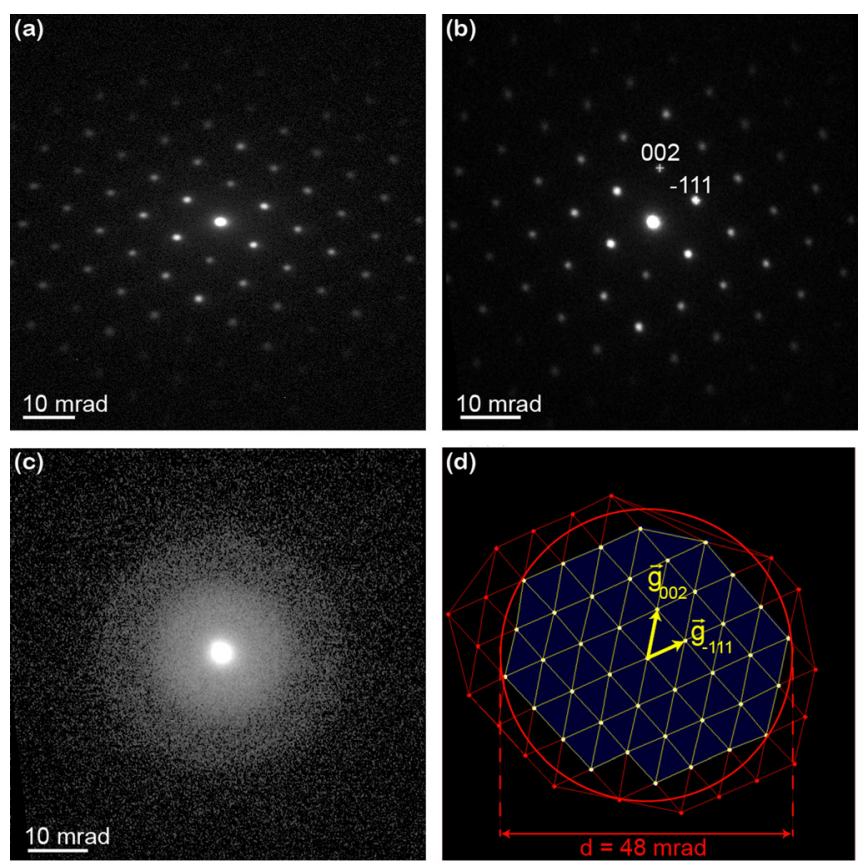

FIG. 1. [110] PED diffraction pattern obtained in silicon (a) without and (b) with projective geometry correction. (c) Electron Nano Beam Diffraction (NBD) amorphous diffraction pattern obtained with projective geometry correction. (d) Delaunay triangulation based strain analysis on Si [110] PED pattern. Distances between direct neighbours (yellow lines) are used $(n=1)$ in a disc shaped transmit centred region with diameter $\mathrm{d}=48 \mathrm{mrad}$ illustrated with the red circle.

this method is able to find a "quasi-kinematic" disc shaped region containing strongly correlated spots and rejecting variable spurious ones.

First, both high and low frequencies are filtered to flatten the image and suppress spurious noise. Then, all the pixel intensity below a threshold value are set to zero; this threshold is set to $I_{\text {mean }}+3 . I_{\text {std }}$ where the mean intensity $I_{\text {mean }}$ and standard deviation $I_{\text {std }}$ are calculated in a diffraction free area of the filtered image. Each spot is indexed by the position $\vec{r}_{i}$ of its intensity centre of mass using ImageJ. ${ }^{13,14} \mathrm{~A}$ Delaunay triangulation ${ }^{15}$ is applied to the ensemble $\vec{r}_{i}$ resulting in a set of Delaunay segments, $\vec{L}_{i j}^{D e l}=\vec{r}_{i}-\vec{r}_{j}$, indexed by their polar coordinates $\left(\theta_{\mathrm{ij}}, \mathrm{l}_{\mathrm{ij}}\right)$. The histogram of the angles $\theta_{\mathrm{ij}}$ modulo $\pi$, build with a binning of $0.5^{\circ}$, shows different classes of direction (indexed by $\mathrm{k}$ ) characterized by their mean angle $\theta_{\mathrm{k}}$ and angle standard deviation $\sigma_{\mathrm{k}}$. The histogram of the $1_{\mathrm{ij}}$ edges associated to each class is built with a binning of 0.5 pixels. The mean length $L_{k}^{(1)}$ and the standard deviation $\mu_{\mathrm{k}}$ of the main class are deduced as well as the lattice vectors $\overrightarrow{g_{i j}}=\left(L_{k}^{(1)}, \theta_{k}\right)$. The method sensitivity is limited by both a systematic error induced by the camera resolution and a statistical error. The former decreases as the measured length increase, and the latter decrease as the measure sample population increases. A trade-off between the segments respective lengths and the total number of segment can be found to enhance the sensitivity of the strain analysis. Therefore, more reliable $\overrightarrow{g_{i j}}=\left(L_{k}^{(n)}, \theta_{k}\right)$ lattice vectors are obtained using lengths satisfying $L_{k}^{(n)}=n L_{k}^{(1)}$.

Both the strain tensor, $\varepsilon_{i j}$, and the rotation tensor, $\Omega_{i j}$, of the crystal are obtained using the method exposed in the Ref. 16. The reciprocal matrix

$$
\mathbf{G}=\left(\begin{array}{ll}
g_{1 x} & g_{1 y} \\
g_{2 x} & g_{2 y}
\end{array}\right)
$$

is built by selecting two lattice vectors among the previously obtained $\overrightarrow{g_{k}}$ (Fig. 1(d)). The components $g_{k x, k y}$ stand for the Cartesian coordinate of $\overrightarrow{g_{k}}$. If $\mathbf{G}_{0}$ is defined as the reciprocal matrix of an unstrained area, the strain and rotation tensor are, respectively, the symmetric component $\varepsilon_{i j}=\left(D_{i j}+D_{j i}\right) / 2$ and the antisymmetric component, $\Omega_{i j}=\left(D_{i j}-D_{j i}\right) / 2$, of the displacement tensor $\mathbf{D}=\mathbf{G}^{-1} \mathbf{G}_{0}-\mathbf{1}$.

The reference sample we have used to benchmark the method has been prepared by Reduced Pressure Chemical Vapour Deposition (RPCVD) on a [001] Si Substrate. It is composed by four $10 \pm 1 \mathrm{~nm}$ SiGe layers with different contents in germanium (respectively, 20\%, 31\%, 38\%, and 45\%) separated by $30 \mathrm{~nm}$ of Si and covered with a $150 \mathrm{~nm}$ Si capping layer. A Focused Ion Beam (FIB) tool (operating at $30 \mathrm{kV}$ and $5 \mathrm{kV}$ ) has been used to provide a $50 \mathrm{~nm}$ thick parallel-sided lamella with reduced surface damage. This sample was specifically designed to benchmark strain stud$\operatorname{ies}^{17}$ as it is possible to simulate the strain expected in TEM using the COMSOL ${ }^{\circledR}$ software. A three-dimensional finite element simulation is performed to take into account the stress relaxation phenomena of the thin foil. The simulated strains are averaged along the [1-10] and [-110] directions. Finally, the strain profile along the [00-1] direction is convoluted with a $4.2 \mathrm{~nm}$ wide electron probe. The simulated $\varepsilon_{\mathrm{xx}}$ strain profile is given in Figure 2(b) (solid black curve).
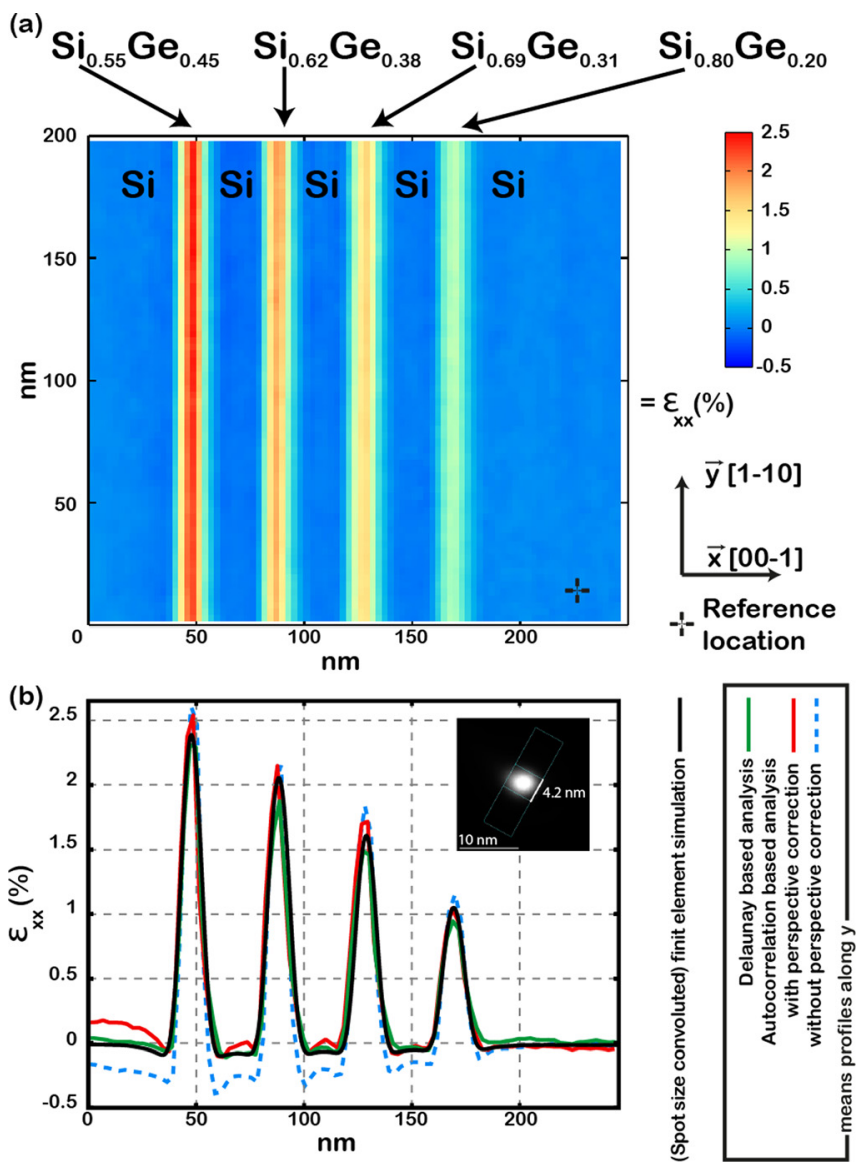

FIG. 2. (a) SiGe Strain mapping with Precession (semi-angle set to $1.44^{\circ}$ ); (b) comparison between the $\varepsilon_{\mathrm{xx}} \mathrm{SiGe}$ strain profile obtained by finite element simulation (solid black curve) and the experimental profiles after different diffraction pattern treatments. 
The proposed method, i.e., perspective correction combined with a Delaunay based treatment, is applied to the PED patterns measured on the reference sample. Results show that the strain sensitivity measured with first-neighbour segments increase until spots outside a transmit beam centred disc-shaped area, with diameter $\mathrm{d}=48 \mathrm{mrad}$, is used (Fig. 1(d)). In addition, the best strain sensibility is reached with second-neighbour segments measurements $(n=2)$. The experimental strain mapping after this data treatment is shown in Figure 2(a). The obtained strain precision is equal to $2.7 .10^{-4}$, as estimated by measuring the rms in 800 silicon contiguous $\varepsilon_{\mathrm{xx}}$ values in an unstrained area. This precision is (i) close to the one obtained by PED measured on highresolution on-axis camera, ${ }^{5}$ (ii) approximately one order of magnitude lower than that achieved by Geometric Phase Analysis (GPA) on non-corrected microscopes. ${ }^{18}$ In the following, our model efficiency is tested by comparing the precision and accuracy obtained on the same PED patterns treated by standard treatments.

For this purpose, the maxima positions are determined using a two-dimensional Gaussian fitting of the diffracted spots, as typically done in the literature. ${ }^{5,8,11,12}$ An autocorrelation of the PED pattern is often implemented ${ }^{11,12}$ to significantly reduce the noise. Therefore, the autocorrelation of each noise filtered diffraction pattern is first calculated. Second, three to four spots positions are fitted with a 2D Gaussian function to construct the reciprocal matrix. Finally, the strain is obtained with respect to an unstrained area reciprocal matrix of reference.

Results shown in Table I prove that the use of a standard data treatment, referred to as "Autocorrelation" in Table I, leads to a drop in strain precision, either with or without prior perspective correction.

The accuracy is here defined as the mean squared error calculated between the simulated (using 3D finite element modelling) and experimental $\varepsilon_{\mathrm{xx}}$ strain profiles after different data treatments (shown in Figure 2(b)). As seen from Table I, the accuracy is also substantially improved using the proposed method. Interestingly, as seen from Figure 2(b), the standard methods fail to predict the slightly compressive strain values in-between the SiGe layers.

Note that an improvement of the PED spatial mapping resolution can be obtained by increasing the beam convergence semi-angle in a TEM equipped with a three condenser lenses system. This leads however to disk-shaped diffraction spots with residual dynamical contrast. In that case, the

TABLE I. Comparison between different data treatments applied to the same set of PED patterns measured on the reference sample.

\begin{tabular}{lcc}
\hline \hline PED analysis & Precision & Accuracy \\
\hline $\begin{array}{l}\text { Delaunay based method } \\
\text { with perspective } \\
\text { correction }\end{array}$ & $2.7 \times 10^{-4}$ & $1.4 \times 10^{-2}$ \\
$\begin{array}{l}\text { Autocorrelation based } \\
\text { method without }\end{array}$ & $3.7 \times 10^{-4}$ & $3.6 \times 10^{-2}$ \\
perspective correction & & \\
$\begin{array}{l}\text { Autocorrelation based } \\
\text { method with perspective } \\
\text { correction }\end{array}$ & $4.0 \times 10^{-4}$ & $2.0 \times 10^{-2}$ \\
\hline \hline
\end{tabular}

positions detection of diffraction disks can be obtained more accurately using the centroid approach available in Image ${ }^{13,14}$ (as it averages the $\mathrm{x}$ and $\mathrm{y}$ coordinates of the pixels within each disk) instead of using the centre of mass method.

The method is also applied to an actual material investigation on InGaAs quantum-well (QW) device ${ }^{19}$ using a noncommon GaAs reference. This high injection velocity material is a promising candidate co,21 $^{20}$ metal Oxide Semiconductor Field Effect Transistor (MOSFET) channels. The device is made of a 10-nm-thick InGaAs QW layer composed of $10 \%$ indium. Special attention was paid to the QW elaboration since misfit dislocations located at the $\mathrm{GaAs} / \mathrm{Si}$ interface are often observed due to the high lattice mismatch. ${ }^{22}$ For this purpose, local GaAs/AlAs/InGaAs/AlAs/ GaAs multi-layers growth has been performed ${ }^{15}$ in an Applied Materials metalorganic chemical vapor deposition (MOCVD) reactor on $300 \mathrm{~mm} \mathrm{Si} \mathrm{(100)} \mathrm{substrates,} \mathrm{as} \mathrm{sche-}$ matically illustrated in Fig. 3(a).

A 80-nm-thick lamella was prepared using FIB tool. The final thinning was performed at $1 \mathrm{keV}$ to reduce implantation and amorphisation. The PED analysis has been carried out on a $160 \times 85 \mathrm{~nm}^{2}$ area using the same experimental conditions as those used for the reference sample (e.g., precession semi-angle of $1.44^{\circ}$ ). The perspective correction and Delaunay based treatment (with $\mathrm{d}=48 \mathrm{mrad}$ and $\mathrm{n}=2$ ) were then applied to the acquired patterns.

As seen from Figure 3(b), results indicate a variation of the $\varepsilon_{\mathrm{xx}}$ strain component in GaAs close to the GaAs/Si interface. Above $100 \mathrm{~nm}$ of GaAs, no significant variation is observed in the $\varepsilon_{\mathrm{xx}}$ strain map. The $\varepsilon_{\mathrm{xx}}$ and $\varepsilon_{\mathrm{yy}}$ strain components fluctuations in the InGaAs QW, and its near environment $( \pm 20 \mathrm{~nm})$, are lower than $0.3 \%$ along the [110] $\mathbf{x}$ direction. These results give evidence of a homogenous strain state of the QW. The $\varepsilon_{\mathrm{yy}}$ strain map exhibits a constant $\sim 1 \%$ tensile strain within the InGaAs QW. This is mostly due to the chemical composition variations and will be addressed in following works, including chemical analysis.

(a)
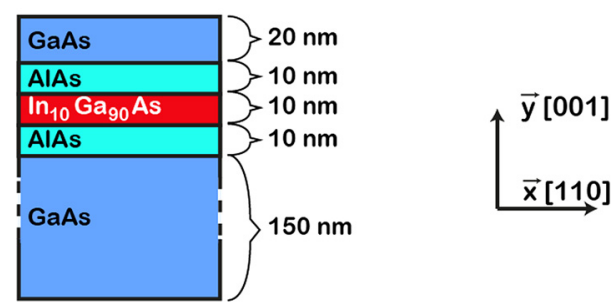

(b)
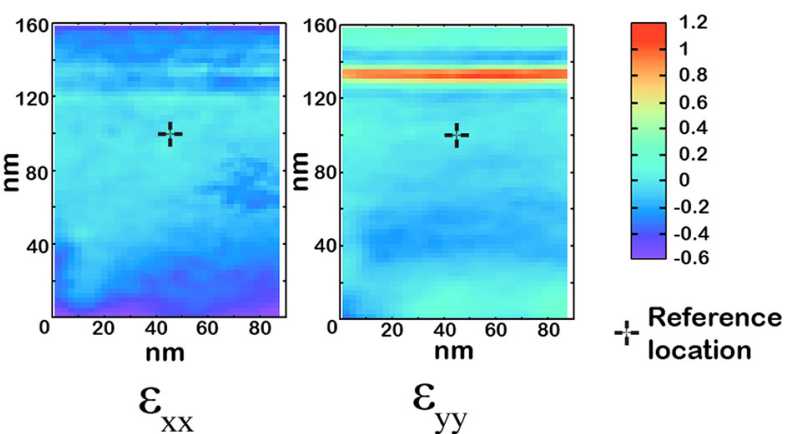

FIG. 3. (a) GaAs/AlAs/InGaAs/AlAs/GaAs multi-layers grown on $300 \mathrm{~mm}$ Si (100) substrates; (b) multi-layers device $\varepsilon_{\mathrm{xx}}, \varepsilon_{\mathrm{yy}}$ strain mapping components obtained with precession (semi-angle set to $1.44^{\circ}$ ). 
This PED analysis is therefore able control that the QW device design efficiently avoids InGaAs layer from being affected negatively by the silicon substrate proximity, which may, e.g., introduce a heterogeneous strain distribution within the QWs. It is also able to distinguish the AlAs, GaAs $0.5 \%$ lattice parameter difference. This was made possible by the combination of a high strain precision $(<0.03 \%)$ and acceptable spatial resolution $(\sim 4 \mathrm{~nm})$ of PED performed in a non-corrected TEM with an off-axis camera. In contrast, other strain techniques performed in the same conventional microscope could not a priori provide such information on the InGaAs QW, e.g., the same level of strain precision $(\sim 0.04 \%)$ with GPA is obtained for a $9 \mathrm{~nm}$ spatial resolution. ${ }^{18}$ The $\varepsilon_{\mathrm{xy}}$ strain component has also been obtained from the strain analysis. It is not shown here as no relevant fluctuation appears due to the quadratic lattice deformation.

In conclusion, the introduction of precession in nanobeam electron diffraction can be used in strain measurements while working with an off axis camera, either on corrected or non-corrected TEMs. Perspective corrected PED patterns associated with a Delaunay based method improve the quality and robustness of strain analysis. This local approach is able to find a "quasi-kinematic" transmitted beam centred disc shaped region containing strongly correlated spots and rejecting variable spurious ones. In the present work, a precision of $2.7 \times 10^{-4}$ with a probe size as small as $4.2 \mathrm{~nm}$ (FWHM) is obtained on a conventional TEM. InGaAs QW device's results show that the GaAs/Si mismatch does not induce in-plane strain fluctuations in the active QW region.

This study was made possible through funding provided by French ANR LABEX MINOS and French ANR AMOS programs. Experiments have been pursued within the Nanocharacterisation Platform of the CEA Grenoble, MINATEC Campus.
${ }^{1}$ R. Vincent and P. A. Midgley, Ultramicroscopy 53, 271 (1994).

${ }^{2}$ A. S. Eggeman and P. A. Midgley, in Advances in Imaging and Electron Physics, edited by P. W. Hawkes (Elsevier Academic Press Inc, San Diego, 2012), Vol. 170.

${ }^{3}$ E. F. Rauch, J. Portillo, S. Nicolopoulos, D. Bultreys, S. Rouvimov, and P. Moeck, Z. Kristallogr. 225, 103 (2010).

${ }^{4}$ L. Palatinus, D. Jacob, P. Cuvillier, M. Klementov, W. Sinkler, and L. D. Marks, Acta Crystallogr., Sect. A 69, 171 (2013).

${ }^{5}$ J. L. Rouviere, A. Béché, Y. Martin, T. Denneulin, and D. Cooper, Appl. Phys. Lett. 103, 241913 (2013).

${ }^{6}$ L. Liao and L. D. Marks, Ultramicroscopy 117, 1-6 (2012).

${ }^{7}$ S. Nicolopoulos, D. Bultreys, and E. Rauch, "Methods and Devices for High Throughput Crystal Structure Analysis by Electron Diffraction," patent No. WO/2010/052289 (15 May 2010).

${ }^{8}$ Beche, A., J. L. Rouviere, L. Clement, and J. M. Hartmann, Appl. Phys. Lett. 95, 123114 (2009).

${ }^{9}$ F O. Faugeras, Three-Dimensional Computer Vision (MIT press, 1993).

${ }^{10}$ E. Peli, J. Opt. Soc. Am. A 7, 2032-2040 (1990).

${ }^{11}$ F. H. Baumann, Appl. Phys. Lett. 104, 262102 (2014).

${ }^{12}$ K. Müller, A. Rosenauer, M. Schowalter, J. Zweck, R. Fritz, and K. Volz, Microsc. Microanal.: Official J. Microsc. Soc. Am., Microbeam Anal. Soc., Microsc. Soc. Can. 18, 995 (2012).

${ }^{13}$ C. A. Schneider, W. S. Rasband, and K. W. Eliceiri, Nat. Methods 9, 671-675 (2012).

${ }^{14}$ S. Bolte and F. P. Cordelières, J. Microsc. 224(3), 213-232 (2006).

${ }^{15}$ O. Gyuhwan and S. Lee, Pattern Recognit. Lett. 23, 1179 (2002).

${ }^{16}$ J. L. Rouvière and E. Sarigiannidou, Ultramicroscopy 106, 1-17 (2005).

${ }^{17}$ A. Béché, J. L. Rouvière, J. P. Barnes, and D. Cooper, Ultramicroscopy 131, 10 (2013).

${ }^{18}$ J. L. Rouviere, in Microscopy of Semiconducting Materials 2007, Springer Proceedings in Physics 120, edited by A. G. Cullis and P. A. Midgley (Springer Netherlands, 2008), pp. 199-202.

${ }^{19}$ R. Cipro, T. Baron, M. Martin, J. Moeyaert, S. David, V. Gorbenko, F. Bassani, Y. Bogumilowicz, J. P. Barnes, N. Rochat, V. Loup, C. Vizioz, N. Allouti, N. Chauvin, X. Y. Bao, Z. Ye, J. B. Pin, and E. Sanchez, Appl. Phys. Lett. 104, 262103 (2014).

${ }^{20}$ S. Takagi, S. H. Kim, M. Yokoyama, R. Zhang, N. Taoka, Y. Urabe, T. Yasuda, H. Yamada, O. Ichikawa, N. Fukuhara, et al., Solid-State Electron. 88, 2-8 (2013).

${ }^{21}$ L. Czornomaz, M. El Kazzi, M. Hopstaken, D. Caimi, P. Mächler, C. Rossel, M. Bjoerk, C. Marchiori, H. Siegwart, and J. Fompeyrine, SolidState Electron. 74, 71-76 (2012).

${ }^{22}$ Y. B. Bolkhovityanov and O. P. Pchelyakov, Open Nanosci. J. 3, 20 (2009). 